\title{
OPN-a Splicing Variant Expression in Non-small Cell Lung Cancer and its Effects on the Bone Metastatic Abilities of Lung Cancer Cells In Vitro
}

\author{
CHENGCHENG HAO ${ }^{1,2}$, YUXIN CUI ${ }^{3}$, MU HU ${ }^{2,4}$, XIUYI ZHI ${ }^{2,4}$, LIJIAN ZHANG $^{5}$, \\ WENBIN LI ${ }^{6}$, WEI WU ${ }^{1,2}$, SHAN CHENG ${ }^{1,2}$ and WEN G. JIANG ${ }^{1,2,3}$ \\ ${ }^{1}$ Department of Biochemistry and Molecular Biology, School of Basic Medical Sciences, \\ Capital Medical University, Beijing, P.R. China; \\ ${ }^{2}$ Beijing Key Laboratory of Cancer \& Metastasis Research, Capital Medical University, Beijing, P.R. China; \\ ${ }^{3}$ Cardiff China Medical Research Collaborative, Cardiff University School of Medicine, Cardiff, U.K.; \\ ${ }^{4}$ Department of Thoracic Surgery, Beijing Xuanwu Hospital, Capital Medical University, Beijing, P.R. China; \\ ${ }^{5}$ Department of Thoracic Surgery, Key Laboratory for Carcinogenesis and Translational Research \\ Ministry of Education, Peking University Hospital, Haidian District, Beijing, P.R. China; \\ ${ }^{6}$ Department of Oncology, Beijing Shijitan Hospital, Capital Medical University, Beijing, P.R. China
}

\begin{abstract}
Background: Osteopontin (OPN) is known to be involved in the development of certain cancers, including non-small cell lung cancer (NSCLC). However, its role in tumour progression remains unclear. The present study investigated the expression and biological impact of the OPN variant, OPN- $a$ in NSCLC. Materials and Methods: OPN-a splicing variant expression in human NSCLC tissues was analyzed by real-time $q P C R$ and immunohistochemistry $(I H C)$, respectively. The impact of $O P N-a$ on cellular functions of lung cancer cells was also evaluated. In addition, an in vitro model was developed for the assessment of interactions between lung cancer cells and bone tissue. Results: The expression of OPN-a was higher in lung cancer tissues compared to normal controls. OPN-a promoted the malignant phenotypes of A549 cells by enhancing celladherent abilities to bone tissues, which could be mediated by the interaction with the cell surface receptor $\alpha v \beta 3$ integrin. Conclusion: OPN-a may represent a bone metastatic factor in human lung cancer, as well as a potential therapy target.
\end{abstract}

Correspondence to: Wen G. Jiang, Cardiff China Medical Research Collaborative, Cardiff University School of Medicine, Heath Park, Cardiff CF14 4XN Cardiff, U.K. E-mail: jiangw@cf.ac.uk and Shan Cheng, Department of Biochemistry and Molecular Biology, School of Basic Medical Sciences, Capital Medical University, Beijing 100069, P.R. China. E-mail: chengs@ccmu.edu.cn

Key Words: Osteopontin, lung cancer, bone metastasis, splicing variant.
Lung cancer is one of the most common causes of malignancy-related death worldwide. Non-small cell lung cancer (NSCLC) accounts for $>80 \%$ of primary lung cancers. Despite improvements in traditional treatments (such as surgical resection, chemotherapy, and radiotherapy), the prognosis for lung cancer patients is still poor with a 5-year overall survival being less than $20 \%$ because of highfrequency metastasis (1). Bone is one of the most common sites for metastasis of lung cancer, and approximately $30-40 \%$ of lung cancer patients show bone metastasis (2). Patients with bone metastasis experience distressful symptoms including bone pain, pathologic fractures, spinal cord and nerve compression, hypercalcemia and decreased mobility, that can seriously affect patients' life quality. Until now the underlying molecular mechanisms that lead to development of bone metastasis in NSCLC are still unclear. Therefore, studying the mechanisms of NSCLC progression and finding more effective biomarkers for an early diagnosis of bone metastasis are crucial for the treatment of this invasive malignancy.

Human osteopontin (OPN), a member of the "small integrin binding ligand N-linked glycoproteins" (SIBLINGs) family, is a 41-75 kDa extracellular matrix phosphoprotein expressed in multiple tissues (3-5). Also, OPN is most abundant in bone (6). During bone remodeling, OPN is produced by the osteoclasts thus contributing to their attachment to the mineral matrix. Previous studies have suggested that OPN expression is particular high in various primary tumors, including lung cancer (7). Furthermore, OPN overexpression is significantly associated with a high incidence of metastasis (8). In bone metastases, OPN seems 
to mediate the interaction between the carcinoma and the bone surface (9). However, the molecular mechanism of OPN tumorigenicity in NSCLC, especially in bone metastasis, is not completely understood.

As a secreted protein with an arginine-glycine-aspartic (RGD) motif and serine-valine-valine-tyrosine-glutamateleucine-arginine (SVVYGLR) sequence, OPN can also modulate cell behavior in autocrine or paracrine manors via their interaction with cell surface receptors such as integrins $(10,11)$. Integrins are a family of transmembrane adhesion receptors, formed by two subunits named $\alpha$ and $\beta$, that interact through non-covalent bindings. As one of the most common integrin heterodimer, $\alpha v \beta 3$ integrin has been identified as a critical receptor for OPN in many biological processes (12). Recent findings indicate that binding and activation of $\alpha \mathrm{v} \beta 3$ integrin by OPN may promote the motility, adhesion and apoptosis of malignant cells $(13,14)$, while the potential biological roles of OPN and $\alpha v \beta 3$ in the pathogenesis of NSCLC bone metastasis have not been fully elucidated.

Several isoforms of OPN transcripts have been identified, including OPN-a (full-length form), OPN-b (lacking exon 5), and OPN-c (lacking exon 4). Among the three OPN isoforms, OPN-a is the most highly expressed in lung cancer cell lines and lung tumors (15). In the present studies, we sought to investigate the protein levels of OPN-a in NSCLC patient tissue and examine the association of this molecule with clinical outcomes. We then examined the biological functions of OPN-a in human lung cancer cell line A549 after in vitro regulation of $\mathrm{OPN}-\mathrm{a}$ expression. Furthermore, we investigated the interaction of OPN-a and $\alpha v \beta 3$ integrin in tumorigenesis and the roles of these two components in the progression of bone metastasis in NSCLC.

\section{Materials and Methods}

Cell lines and human lung cancer specimen. A549, a human lung adenocarcinoma cell line, was obtained from the American Type Culture Collection (ATCC, Manassas, VA, USA). Cells were routinely maintained with Dulbecco's modified Eagle medium (DMEM)-F12 supplemented with $10 \%$ fetal calf serum and $1 \mathrm{x}$ penicillin/streptomycin. A total of 182 lung tissue samples (92 were normal lung tissue and 90 were lung cancer tissue) from Peking University Hospital and another 10 fresh-frozen NSCLC tissues along with matched normal tissues from Xuanwu Hospital of Capital Medical University were obtained from NSCLC patients who received curative resection. These tissues were collected immediately after surgical resection and stored at $-80^{\circ} \mathrm{C}$ until use. Clinicopathological factors, including age, sex, histological types of tumors, TNM stage, and lymph node metastasis were recorded in the patients' database.

$R N A$ preparation and real-time quantitative polymerase chain reaction ( $q P C R)$. RNA extraction, reverse transcription (RT) and qPCR were performed as described previously. OPN-a qPCR primers as follow: sense: 5'-ACAACAAATACCCAGATGCT-3'; antisense: 5'-GTCATTGGTTTCTTCAGAGG-3'. Z-sequence on qPCR primers is 5'-ACTGAACCTGACCGTACA-3', which is complementary to the universal $\mathrm{Z}$ probe used for the QPCR (TCS Biological Ltd., Oxford, UK). Real-time QPCR conditions were $95^{\circ} \mathrm{C}$ for $15 \mathrm{~min}$, followed by 60 cycles at $95^{\circ} \mathrm{C}$ for $20 \mathrm{~s}, 55^{\circ} \mathrm{C}$ for $30 \mathrm{~s}$ and $72^{\circ} \mathrm{C}$ for $20 \mathrm{~s}$.

Immunohistochemical (IHC) staining. Frozen sections of lung tissues were cut at a thickness of $4 \mu \mathrm{m}$. After incubation with $5 \%$ Bull Serum Albumin blocking solution for $30 \mathrm{~min}$, the sections were probing with the OPN antibody (1:200) (sc-10591, Santa Cruz, CA, USA) together with a negative control without primary antibody. Following extensive washing, sections were incubated for $30 \mathrm{~min}$ with the relevant secondary antibody (ZSGB Biotechnology, Beijing, China). Finally, Section colour was developed with the diaminobenzidine (DAB) chromogen (Cell Signal Technology, Danvers, MA, USA) and then were observed using light microscopy (BX43, Olympus, Tokyo, Japan).

Construction of OPN plasmids and cell transfection. OPN-a cDNA was cloned into GV141 vector by Genechem Company (Shanghai, China) to establish the OPN-a expression construct. The shRNA plasmid targeting OPN was constructed by Genechem Company (Shanghai, China) using vector GV248. A549 cells were transfected with OPN-a construct and anti-OPN-shRNA transgene using NeofectTM DNA transfection reagent (Neofect Biotech, Beijing, China). OPN expression was verified by western blotting after cells were transfected for $48 \mathrm{~h}$.

Western blotting. To detect the expression level of OPN in the NSCLC cells, confluent cells were pelleted and then lysed using a lysis buffer. These proteins were separated using 10\% Sodium dodecyl sulfate-polyacrylamide gel electrophoresis (SDS-PAGE) and blotted on to nitrocellulose membrane. Membrane was treated with $5 \%$ milk to block non-specific proteins prior to probing with anti-OPN primary antibody (1:500) (sc-10591, Santa Cruz) and antiGAPDH antibody $(1: 1,000)$ (sc-32233, Santa Cruz), followed by peroxidase conjugated secondary antibodies. Protein bands were visualized using a chemiluminescence (ECL) detection system (Millipore, Watford, Hertfordshire, UK).

In vitro cell growth assay. Cells were plated into 96-well plates at density of 2,000 cells/well. The cell viability was then evaluated by a nonradioactive cell counting kit (CCK8, Dojindo, Kamimashikigun, Kumamoto, Japan) assay according to manufacturer's instruction. Briefly after incubation for $0,1,2,3$ and 4 days respectively, the cell viability was determined at a wavelength of $450 \mathrm{~nm}$ using a spectrophotometer (BioTek, Winooski, VT, USA).

Wounding/migration assay. The cells were seeded at a density of $6 \times 10^{5}$ per well into a 12 -well plate and allowed to reach confluence. The monolayer of cells was then scraped with a fine gauge needle to create a wound. The movement of cells to close the wound was recorded using an inverted microscope. Images were captured at the time intervals of $0,6,12$ and $24 \mathrm{~h}$.

Cell matrix adhesion assay. A 96-well plate was precoated with $5 \mu \mathrm{g} / \mathrm{well}$ of Matrigel (BD Biosciences, Oxford, UK) and allowed to dry. Following rehydration by serum-free medium, 
$2 \times 10^{4}$ cells were seeded to each well. After $40 \mathrm{~min}$ of incubation, non-adherent cells were washed off using PBS buffer. The remaining cells were fixed with $4 \%$ formalin, stained with $0.5 \%$ (weight/volume) crystal violet and quantified under a microscope.

In vitro invasion assay. Transwell inserts (upper chamber) with $8 \mu \mathrm{m}$ pore size were coated with Matrigel and air dried. Following rehydration, cells were seeded at a density of $2 \times 10^{4}$ per insert and allowed to invade for 2 days. After incubation, cells that had migrated through the matrix and adhered to the other side of the inserts were fixed in $4 \%$ formalin, stained with $0.5 \%$ (weight/volume) crystal violet, and counted under a microscope.

Interaction with bone matrix. The femurs were extracted from 6month-old minipigs, then after being sterile with $75 \%$ alcohol, the femur heads were made into bone slices with diameter of $20 \mathrm{~mm}$ and thickness of $4 \mathrm{~mm}$. In order to obtain better cultivation conditions for accurate observation, the bone slides were polished by an apparatus to produce a smooth surface (Figure 4A-1). A549 cells were pre-stained with a fluorescent indicator according to its instruction and then seeded onto these bone sections (Figure 4A-2). After incubation of A549 cells on the bone sections for $1 \mathrm{~h}$, nonadherent cells were washed with PBS. The remaining cells were fixed with $4 \%$ formalin and were qualitatively assessed by a fluorescence microscope (Nikon, ECLIPSE, Ti-U, Japan).

Statistical analysis. Statistical analysis was performed using the SPSS software package (Version 19.0 SPSS, Chicago, IL, USA). Briefly, the Normal-distributed data were assessed using non-paired (two-sided) Student's $t$-test (for two groups), one-way ANOVA test (for multiple groups). While Data did not follow normal distribution were assessed using Mann-Whitney $U$-test or Kruskal-Wallis test. OPN mRNA values obtained in the QPCR study are given as median transcript copy number per $50 \mathrm{ng}$ of $\mathrm{RNA} \pm \mathrm{SD}$. A $p$-value $<0.05$ was defined as statistically significant.

\section{Results}

Association of OPN-a expression with clinical features of NSCLC tissue. The expression pattern of OPN-a was detected in 182 tissue specimens from NSCLC patients using QPCR. The associations between clinicopathologic features and the expression levels of OPN-a were shown in Table I. Statistical analysis indicated that OPN-a mRNA in primary lung cancer tissues was significantly higher than that in the paired normal tissues $(p=0.036)$. Furthermore, the elevated level of OPN-a appeared to be more frequent in samples with high tumour stage, positive lymph node metastasis and high TNM stage, although statistical analysis did not show significance among these groups (Table I).

In addition, the expression pattern of OPN at the protein level showed the similar results in the IHC assay. NSCLC specimens showed markedly higher cytoplasmic protein expression of OPN, while the paired normal tissues consistently displayed weaker or undetectable immunostaining (Figure 1).
Table I. Relationship between OPN-a expression and clinical pathological parameters in tissue from lung cancer patients.

\begin{tabular}{lccc}
\hline Variable & $\mathrm{N}$ & Median (IQ range) & $p$-Value \\
\hline $\begin{array}{l}\text { Tissue paired samples } \\
\quad\end{array}$ & 81 & $866(135,15764)$ & 0.036 \\
$\quad \begin{array}{l}\text { Paired tumor } \\
\text { Paired normal }\end{array}$ & 81 & $67(9,465)$ & \\
Smoking history & & & \\
$\quad$ Yes & 51 & $1612(51,10394)$ & 0.97 \\
$\quad$ No & 27 & $378(177,16851)$ & \\
Histology & & & \\
$\quad$ Adenocarcinoma & 41 & $1612(46,29340)$ & 0.48 \\
$\quad$ Squamous carcinoma & 30 & $1135(205,10779)$ & \\
T stage & & & \\
T1 & 10 & $371(118,11627)$ & 1.00 \\
$\quad$ T4 & 12 & $1670(25,9767)$ & \\
Lymphy node status & & & \\
$\quad$ N0 & 36 & $463(26,15621)$ & 0.65 \\
N2+3 & 41 & $1727(208,14189)$ & \\
TNM staging & & & \\
TNM1 & 24 & $440(36,17492)$ & 0.95 \\
TNM2+3+4 & 53 & $1404(177,14189)$ & \\
Differentiation & & & \\
High-Medium & 5 & $1612(638,19793)$ & \\
Medium & 36 & $434(118,16063)$ & 0.083 \\
Medium-Low & 14 & $682(17,4675)$ & 0.34 \\
Low & 11 & $3955(165,47452)$ & 0.16 \\
\hline
\end{tabular}

Effects of OPN-a on in vitro functions of A549 cells. The expression level of OPN was considerably increased in the OPN-a-overexpressing cells (Figure 2A) and reduced OPN expression markedly in the OPN-knockdown cells in A549 (Figure 2F). Compared to control groups, overexpression of OPN-a remarkably promoted malignant phenotype of human A549 cells, including cell proliferation, migration, adhesion and invasion. ( $p<0.01$, respectively) (Figure 2B, C, D and $\mathrm{E})$, whereas knockdown of OPN expression resulted in a significant reduction in growth ability of A549 cells (Figure $2 \mathrm{G})(p<0.05)$. Cell adhesion, invasion and migration were also significantly inhibited respectively $(p<0.01)$ (Figure $2 \mathrm{H}$, I and J).

Effects of potential interaction between OPN and $\alpha v \beta 3$ integrin on the malignant phenotypes of A549 cells. By depleting OPN with a specific antibody adding in medium, cell growth, migration, adhesion, and invasion were dramatically inhibited over $85 \%, 60 \%, 60 \%$ and $70 \%$ respectively, compared with the control groups (adding IgG- neu Ab) $(p<0.01)$ (Figure 3). The similar results were observed that when the integrin $\alpha v \beta 3$-neutralizing antibody was added to the culture medium of A549 cells, the cell growth, migration, adhesion and invasion were reduced to $70 \%, 50 \%, 40 \%$ and $45 \%$ respectively, compared to the control groups (adding IgG-neu Ab) $(p<0.01)$ (Figure 3). 


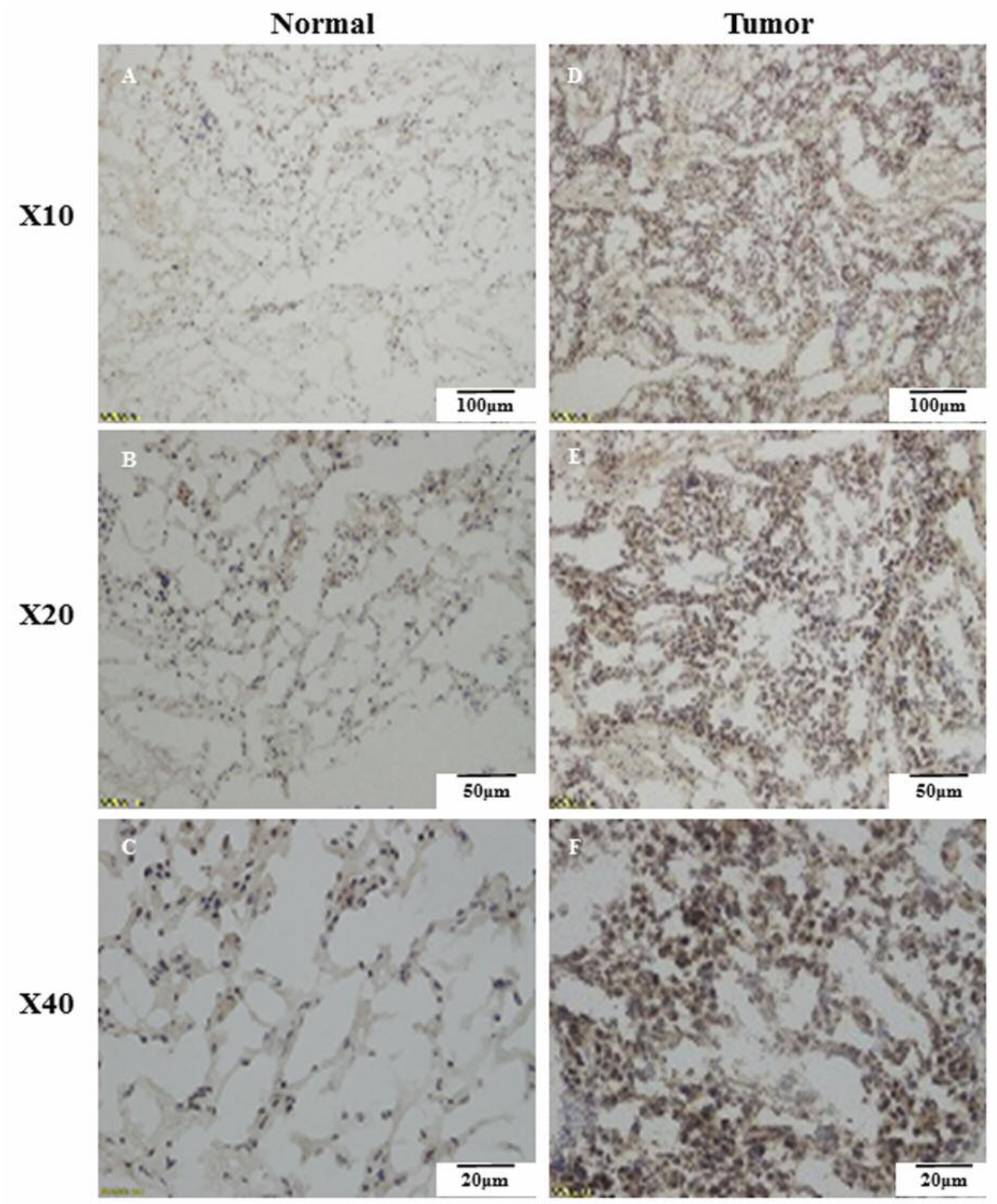

Figure 1. Representative IHC staining of OPN in normal and lung carcinoma tissue sections. A, B, C: Normal tissue were OPN negative (SP $\times 100$, 200, 400). D, E, F: Strong OPN cytoplasmic staining in lung cancer cells (SP $\times 100,200,400)$.

The results suggested that secreted OPN played the main roles in the promotion of the malignant phenotypes of A549 cells, and mostly dependent on the interaction with $\alpha v \beta 3$ integrin receptor.
Effects of interaction between $O P N$ and $\alpha v \beta 3$ integrin on $A 549$ cells attachment to bone matrix. Overexpression of OPN-a significantly promoted the cell adherence to the bone slices $(p<0.01)$ (Figure 4B), while knockdown of OPN significantly 
A

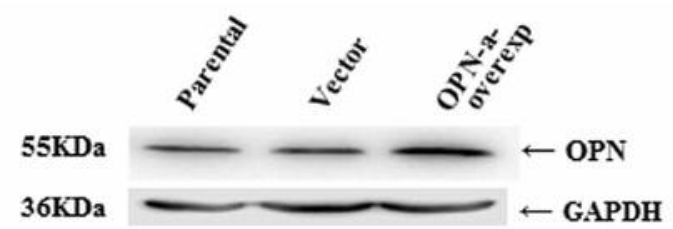

B

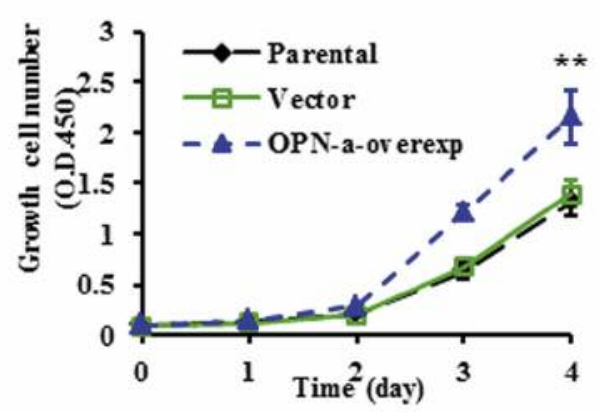

C

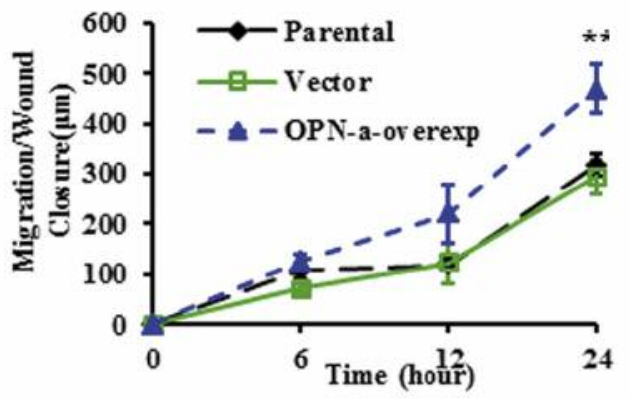

D

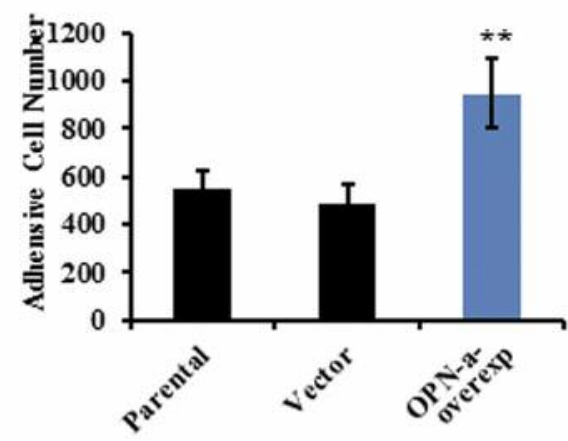

E

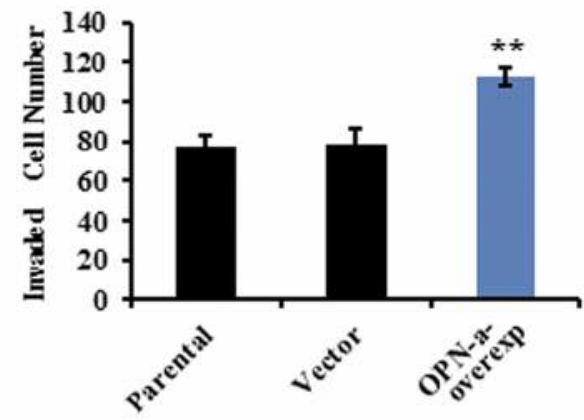

F

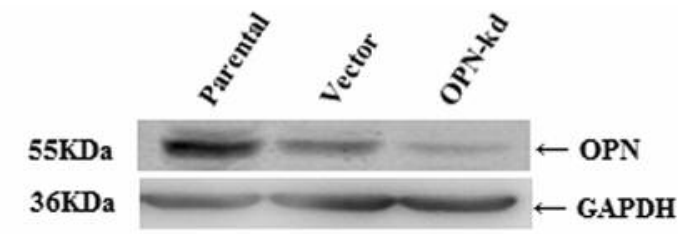

G

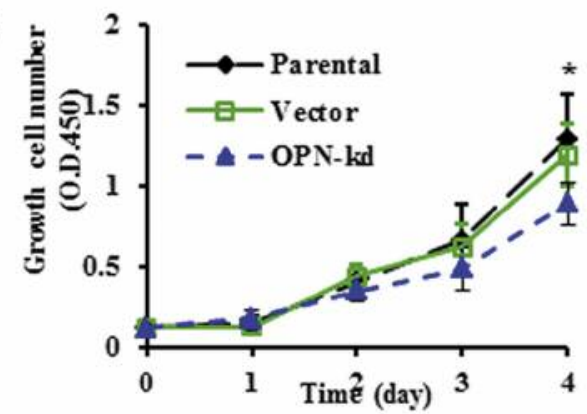

H

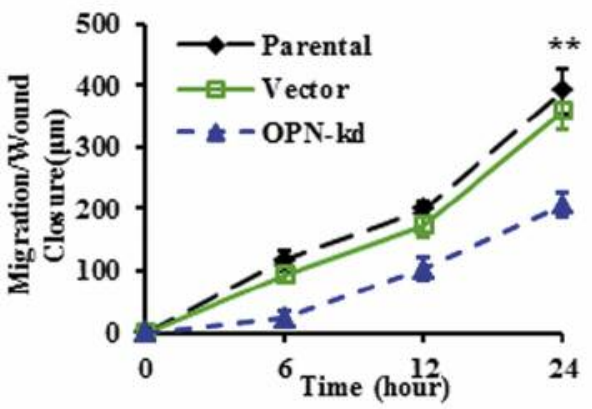

I

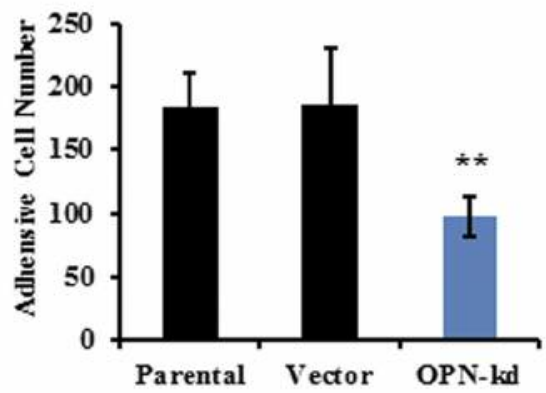

J

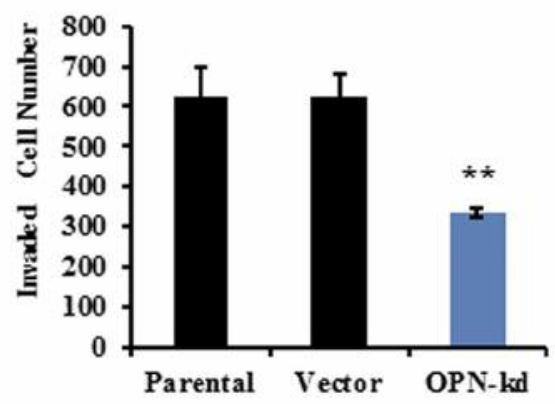

Figure 2. Functional analysis of OPN- $a$ in A549 cells. A: Validation of ectopic overexpression of OPN in A549 cells by western blotting. B: OPNa overexpression promoted A549 cell growth over the experimental time points compared to the control cells. C: OPN-a overexpression in A549 promoted cellular migration. D: OPN-a overexpression enhanced cell matrix adhesion compared with control. E: Significant increase of in vitro Matrigel invasion in OPN-a-overexpressing cells. F: Knockdown of OPN in A549 cells as showed by western blotting. G, H, I, J: Knockdown of OPN in A549 cells reduced the cellular growth, migration, adhesion and Matrigel invasion, respectively. ${ }^{*} p<0.05, * * p<0.01$. 
A

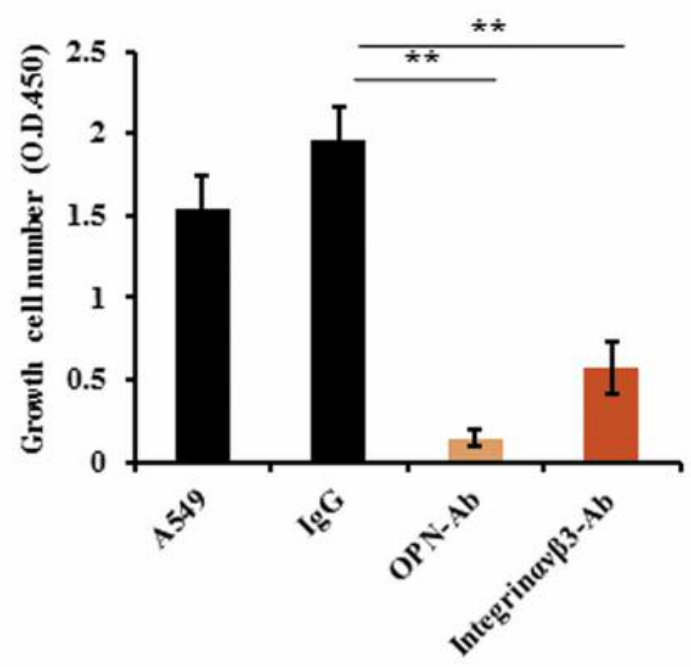

C

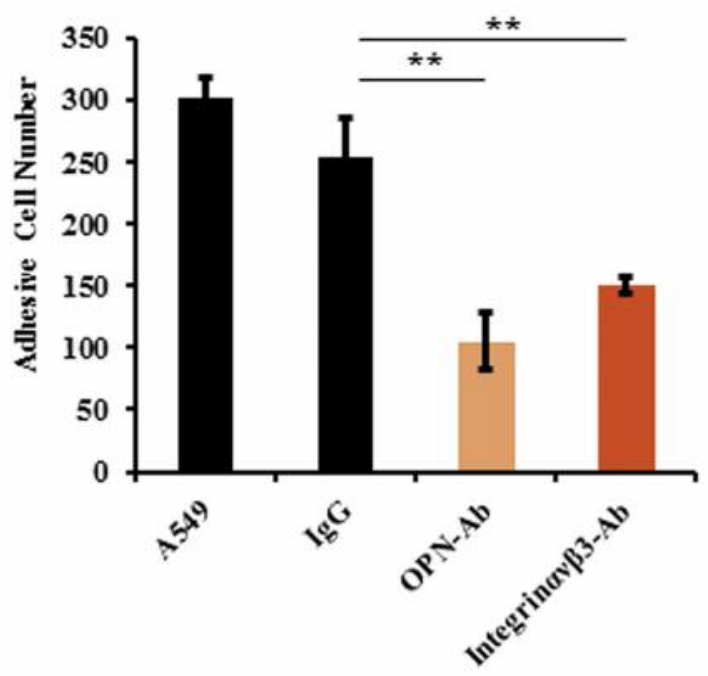

B

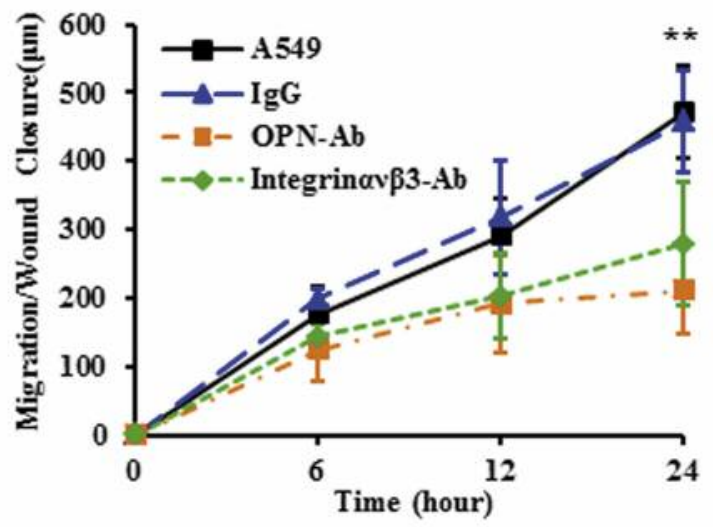

D

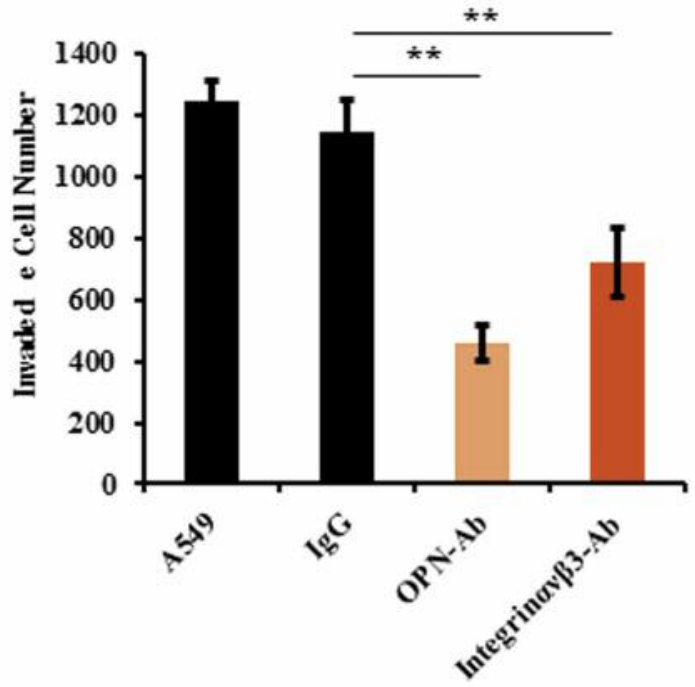

Figure 3. Involvement of both secreted OPN and $\alpha v \beta 3$ integrin in functions of A549 cells. We supplemented the same concentration (20 $\mu \mathrm{g} / \mathrm{ml})$ of $O P N$-neutralizing antibody and $\alpha v \beta 3$ integrin-neutralizing antibody to the media of cultured $A 549$ cells respectively and investigated the properties of A549 cells. Functions of A549 cells including growth (A), migration (B), adhesion $(C)$ and invasion (D) were dramatically inhibited in OPNneu $\mathrm{Ab}$ and $\alpha v \beta 3$ integrin-neu Ab groups respectively compared to WT and IgG-neu Ab control groups $(p<0.01)$. In addition, the blocking effects of the OPN-neu Ab were more significant than those groups with the integrin $\alpha v \beta 3-n e u A b(p<0.01)$.

inhibited the A549 cells attachment to bone slices $(p<0.01)$ (Figure 4C). Following incubation of cells with OPNneutralizing antibody and $\alpha v \beta 3$ integrin-neutralizing antibody respectively, the adhesion properties of A549 cells to bone matrix were strongly inhibited and the blocking effect by OPN antibody was stronger than that by $\alpha v \beta 3$ integrin antibody (compared with control group, $p<0.01$, respectively) (Figure 4D).

\section{Discussion}

OPN is one of the known factors that have been considered to be correlated with the progression and severity of certain cancer types, including lung, breast, colon and prostate (16-19).
It has been reported that high OPN expression in the primary tumor is associated with early metastasis and poor clinical outcome. In the present study, we have showed that OPN-a is significantly overexpressed in NSCLC tissues when compared to matched normal lung tissues. Additionally, we focused on the role of OPN-a in mediation of malignant phenotype of NSCLC cells. Our present results demonstrate that overexpression of OPN-a is linked to the elevation of growth, adhesion, motility and invasion properties of A549 cells in vitro. On the contrary, when OPN was silenced, there was a significant reduction of the cellular growth, adhesion, motility and invasion properties of the A549 cells. Taken together, these results demonstrate the pro-cancer role of OPN-a in A549 cells. 
A-1
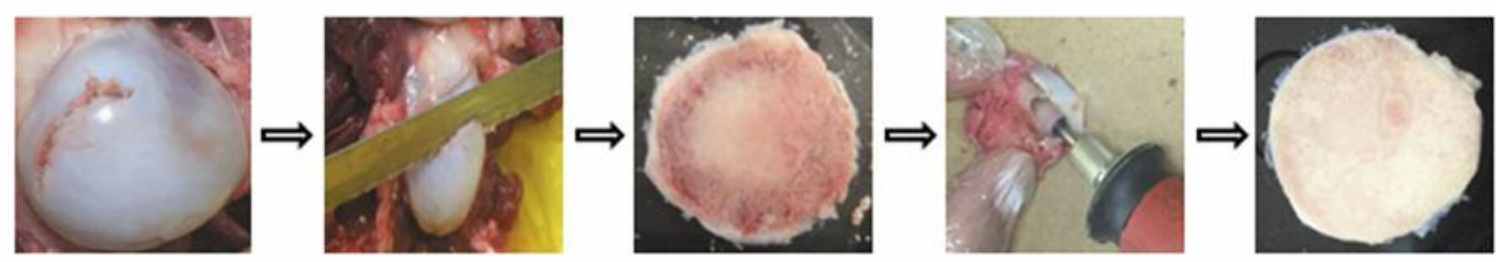

A-2
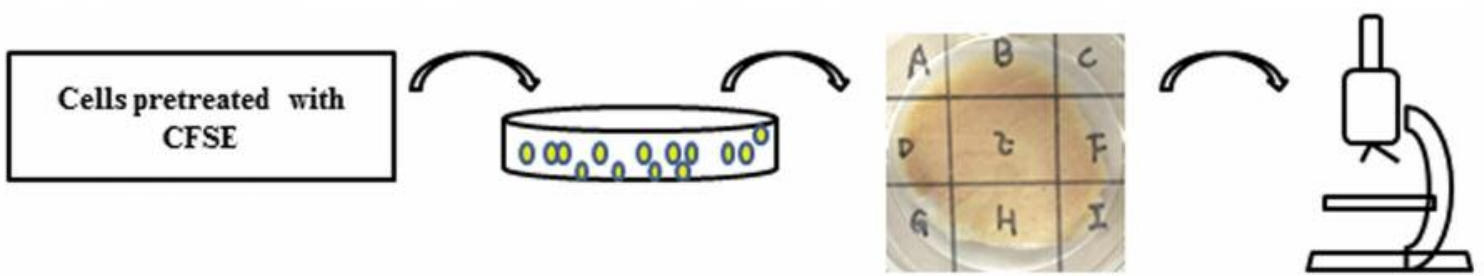

B

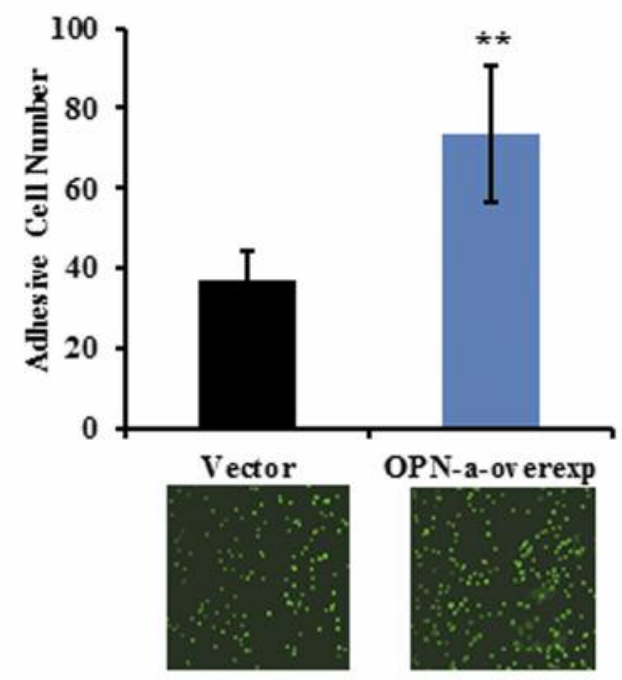

C

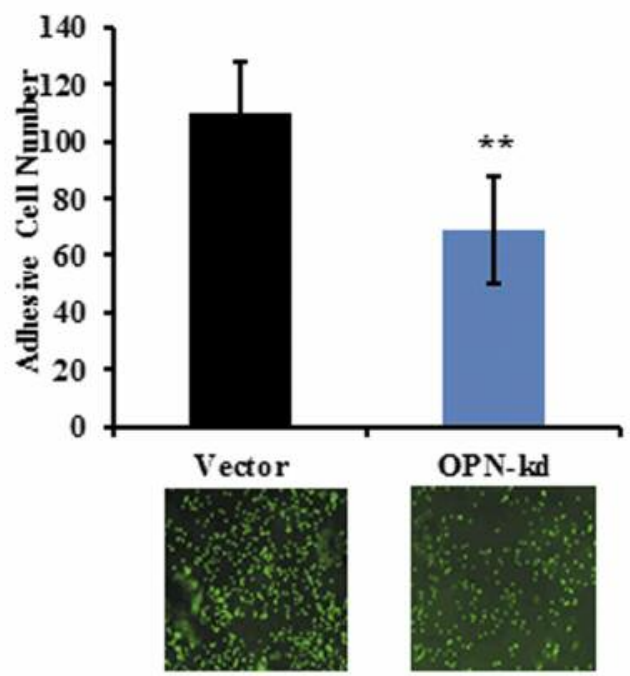

D

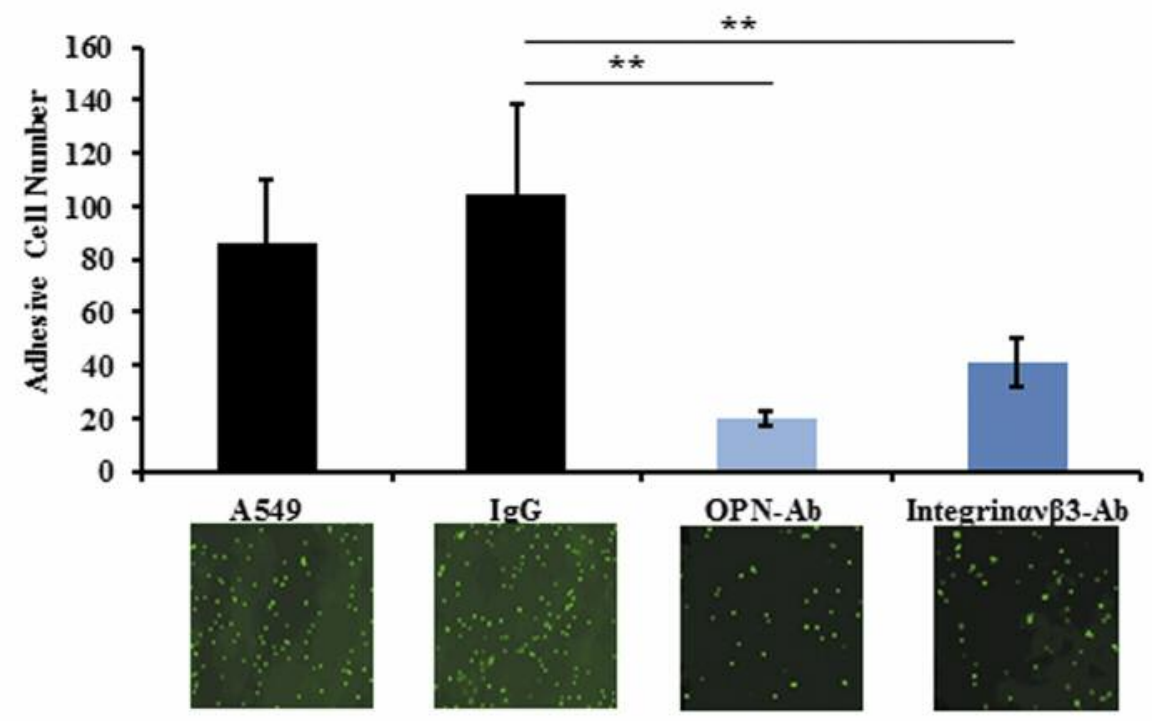

Figure 4. Effect of OPN and $\alpha v \beta 3$ integrin on adhesion of A549 cells to bone matrix. A-1, A-2: The femur heads from minipigs were cut into bone slices with diameter of $20 \mathrm{~mm}$ and thickness of $4 \mathrm{~mm}$. And bone slices were polished by a related tool for a smooth surface. The fraction of attached cells was stained by a fluorescent indicator and then and visually counted. B, C: OPN could specifically affect adhesion of A549 cells to bone slices. D: By depleting $O P N$ with antibody adding in medium, the secreted OPN-induced cell attachment to bone matrix was significantly inhibited in A549. After the interaction between secreted OPN and membrane $\alpha v \beta 3$ integrin was blocked, the OPN-induced cell malignant phenotype was partially inhibited. 
OPN has been mainly studied as a secreted protein involved in cancer progression and metastasis. For example, studies show OPN is often present at high levels in the circulation of patients with metastatic cancers and in tumors with increased metastatic potential $(8,20)$. However, recent reports indicate that intracellular OPN can also play specific roles inside the cells, such as cell motility, cytoskeletal rearrangement, and mitosis by physical interaction with pololike kinase-1 (Plk-1) $(21,22)$. In order to determine the significance of the secreted OPN (sOPN) in lung cancer progression and metastasis, the effects of sOPN on the malignant phenotypes of A549 cells were investigated by adding the OPN-neutralizing antibody in the A549 cell media. Blocking of sOPN resulted in reduced cellular growth, migration, adhesion and invasion suggesting the actual roles of sOPN in the promotion of the malignant phenotypes of A549 cells.

$\alpha v \beta 3$ Integrin was also considered in our research because it is the main membrane receptor of sOPN and an important heterodimer in integrin family. At the stages of cancer progression, $\alpha v \beta 3$ integrin is activated and then interacts with OPN, thus providing the necessary signals for the adhesion, migration, and cell survival. For example, cancer cells with aberrant $\alpha v \beta 3$ integrin expression can increase the chemotactical migration ability towards OPN (23). When OPN binds to the $\alpha v \beta 3$ integrin on cell surface, breast cancer cells are induced a great resistance to apoptosis (24). In our study, the results confirmed that although the blocking is not as efficient as that of OPN antibody, the $\alpha v \beta 3$ integrin antibody also remarkably inhibits the malignant properties of A549 cells. Thus, OPN appears to function in promoting the malignant phenotypes of A549 probably through its interaction with $\alpha v \beta 3$ integrin receptor.

The mechanisms that regulate the movement of the primary tumour to the bone have not been elucidated as yet, however the interaction between cancer cells and bone matrix is thought to be an early step in bone metastasis formation. It has been suggested that OPN and $\alpha v \beta 3$ integrin interaction may mediate many of the activities necessary for this initial step in the bone metastasis of malignancies. A number of studies showed that overexpression of OPN were determinative of a high incidence of bone metastases in breast cancer (25). Likewise, in prostate cancer, activation of $\alpha v \beta 3$ integrin allows cancer cells to adhere and migrate to bone matrix at early stages of skeletal metastasis (26). In order to understand the effects of OPN and $\alpha v \beta 3$ integrin on early steps bone colonization, we assessed A549 cells attachment (after OPN overexpression and knockdown in cells, respectively) to minipig bone slices. Interestingly, we observed that the A549 cells following OPN silencing displayed a significant inhibition of bone matrix attachment. In contrast, over expression of OPN-a in A549 cells increased their preferential migration to the bones. Moreover, after treated with OPN antibody or $\alpha v \beta 3$ integrin antibody, A549 cells showed a strikingly reduced attachment ability to bone slices. Thus, the results suggest that when lung cancer cells invade in bone, the binding of OPN and $\alpha v \beta 3$ integrin could play a vital role in tumour cell adhesion at bone matrix. We established a new model of bone metastasis formation with pig bone for the first time, which presents certain advantages including simple operation and better simulation. However, this model could still have space to be improved, especially because the operation on the bone slices may destroy the bone marrow endothelial cells, thus disrupting the interactions between the cancer cells and the bone microenvironment.

Collectively, our results have clearly demonstrated the clinical value of OPN-a in human non-small cell lung cancer as a potential target for therapy and a potential prognostic factor. The study has also revealed the importance of OPN-a in the aggressiveness of lung cancer cells with a particular relevance to bone metastasis related cell functions of lung cancer cells. Presently, we are developing a test to evaluate the circulating levels of OPN in patients with lung cancer in order to establish if plasma levels of OPN can also be a prognostic/diagnostic factor for these patients and hopefully to develop a clinically deliverable test in the regards. Indeed, circulating OPN in patients with breast cancer (27), liver cancer (28), colorectal cancer (29), squamous cell carcinoma (30), ovarian cancer (31) and in non-cancer related medical conditions such as systemic lupus erythematomatus (32) and in autoimmune encephalomyelitis (33) have been reported to have clinical relevance. In lung cancer, this has also been tested, plasma OPN prior and after radiation therapy has been suggested to be an additional prognostic marker (34). We view this type of test, if developed successfully, would make it a convenient and reliable tool in the clinical settings. However, significant challenges exist including deciphering the variant and cleavage OPNs in the circulation (35). Furthermore, we are currently exploring the expression pattern of the OPN protein, both other as a family and for other variants, namely OPN-b and OPN-c, in lung cancer and explore the biological significant of these variant, alone or in combination in the disease progression of NSCLC. Finally, it would be reasonable suggestion that OPN-a should be continuously explore in the context of bone metastasis given the intimate link between OPN-a and the bone metastatic models using here. We are currently identifying a large clinical cohort of lung cancer with bone metastasis information and hopefully to further establish this link in a clinical setting.

In conclusion, our data suggest that higher levels of OPNa are associated with poorer clinical outcome of lung cancer patients. The in vitro cell function results indicate that OPNa acts as an oncogene in lung cancer cells. Moreover, $\alpha v \beta 3$ integrin is involved in the OPN-a-mediated malignant behaviors of lung cancer cells. The interaction of OPN and 
$\alpha v \beta 3$ integrin may play a vital role in adhesion of lung cancer cells to the bone matrix, which is probably one of the early steps of the bone metastasis in lung cancer. Therefore, OPN-a may have potential clinical applications as a biomarker for prognosis and diagnosis, and a novel therapeutic target in lung cancer.

\section{Acknowledgements}

This work was funded by the National Natural Science Foundation of the People's Republic of China (81572704), Cancer Research Wales, Cardiff University China Medical Scholarship and the Albert Hung Foundation. The Authors would like to thank Peking University Hospital and Xuanwu Hospital of Capital Medical University for supplying lung cancer tissue samples. They also thank Valentina Flamini for her valuable suggestions for the manuscript.

\section{References}

1 Brody H: Lung cancer. Nature 513: S1, 2014.

2 Mundy GR: Metastasis to bone: causes, consequences and therapeutic opportunities. Nature reviews Cancer 2: 584-593, 2002

3 Bellahcene A, Castronovo V, Ogbureke KU, Fisher LW and Fedarko NS: Small integrin-binding ligand N-linked glycoproteins (SIBLINGs): multifunctional proteins in cancer. Nature reviews Cancer 8: 212-226, 2008.

4 Christensen B, Nielsen MS, Haselmann KF, Petersen TE and Sorensen ES: Post-translationally modified residues of native human osteopontin are located in clusters: identification of 36 phosphorylation and five O-glycosylation sites and their biological implications. Biochem J 390: 285-292, 2005.

5 Christensen B, Petersen TE and Sorensen ES: Post-translational modification and proteolytic processing of urinary osteopontin. Biochem J 411: 53-61, 2008.

6 Denhardt DT and Noda M: Osteopontin expression and function: role in bone remodeling. J Cell Biochem Suppl 30-31: 92-102, 1998.

7 Fedarko NS, Jain A, Karadag A, Van Eman MR and Fisher LW: Elevated serum bone sialoprotein and osteopontin in colon, breast, prostate, and lung cancer. Clin Cancer Res 7: 4060-4066, 2001.

8 Bramwell VH, Doig GS, Tuck AB, Wilson SM, Tonkin KS, Tomiak A, Perera F, Vandenberg TA and Chambers AF: Serial plasma osteopontin levels have prognostic value in metastatic breast cancer. Clin Cancer Res 12: 3337-3343, 2006.

9 Bourguignon LY, Zhu H, Shao L, Zhu D and Chen YW: Rhokinase (ROK) promotes CD44v(3,8-10)-ankyrin interaction and tumor cell migration in metastatic breast cancer cells. Cell Motility Cytoskeleton 43: 269-287, 1999.

10 Scatena M, Liaw L and Giachelli CM: Osteopontin: a multifunctional molecule regulating chronic inflammation and vascular disease. Arteriosclerosis Thrombosis Vascular Biol 27: 2302-2309, 2007.

11 Yokosaki Y, Matsuura N, Sasaki T, Murakami I, Schneider H, Higashiyama S, Saitoh Y, Yamakido M, Taooka Y and Sheppard D: The integrin alpha(9)beta(1) binds to a novel recognition sequence (SVVYGLR) in the thrombin-cleaved amino-terminal fragment of osteopontin. J Biol Chem 274: 36328-36334, 1999.
12 Liaw L, Lindner V, Schwartz SM, Chambers AF and Giachelli CM: Osteopontin and beta 3 integrin are coordinately expressed in regenerating endothelium in vivo and stimulate Arg-Gly-Aspdependent endothelial migration in vitro. Circulation Res 77: 665-672, 1995.

13 Allan AL, George R, Vantyghem SA, Lee MW, Hodgson NC, Engel CJ, Holliday RL, Girvan DP, Scott LA, Postenka CO, AlKatib W, Stitt LW, Uede T, Chambers AF and Tuck AB: Role of the integrin-binding protein osteopontin in lymphatic metastasis of breast cancer. Am J Pathol 169: 233-246, 2006.

14 Cui R, Takahashi F, Ohashi R, Gu T, Yoshioka M, Nishio K, Ohe Y, Tominaga S, Takagi Y, Sasaki S, Fukuchi Y and Takahashi K: Abrogation of the interaction between osteopontin and alphavbeta3 integrin reduces tumor growth of human lung cancer cells in mice. Lung Cancer 57: 302-310, 2007.

15 Goparaju CM, Pass HI, Blasberg JD, Hirsch N and Donington JS: Functional heterogeneity of osteopontin isoforms in nonsmall cell lung cancer. JThoracic Oncol 5: 1516-1523, 2010.

16 Hsieh IS, Huang WH, Liou HC, Chuang WJ, Yang RS and Fu WM: Upregulation of drug transporter expression by osteopontin in prostate cancer cells. Mol Pharmacol 83: 968-977, 2013.

17 Lin Q, Guo L, Lin G, Chen Z, Chen T, Lin J, Zhang B and Gu $\mathrm{X}$ : Clinical and prognostic significance of OPN and VEGF expression in patients with non-small-cell lung cancer. Cancer Epidemiol 39: 539-544, 2015.

18 Anborgh PH, Caria LB, Chambers AF, Tuck AB, Stitt LW and Brackstone M: Role of plasma osteopontin as a biomarker in locally advanced breast cancer. Am J translational Res 7: 723-732, 2015.

19 Ng L, Wan TM, Lam CS, Chow AK, Wong SK, Man JH, Li HS, Cheng NS, Pak RC, Cheung AH, Yau TC, Lo OS, Foo DC, Poon JT, Poon RT, Pang RW and Law WL: Post-operative plasma osteopontin predicts distant metastasis in human colorectal cancer. PloS one 10: e0126219, 2015.

20 Ramankulov A, Lein M, Kristiansen G, Loening SA and Jung $\mathrm{K}$ : Plasma osteopontin in comparison with bone markers as indicator of bone metastasis and survival outcome in patients with prostate cancer. The Prostate 67: 330-340, 2007.

21 Junaid A, Moon MC, Harding GE and Zahradka P: Osteopontin localizes to the nucleus of 293 cells and associates with pololike kinase-1. AmJ Physiol Cell Physiol 292: C919-926, 2007.

22 Zohar R, Suzuki N, Suzuki K, Arora P, Glogauer M, McCulloch $\mathrm{CA}$ and Sodek J: Intracellular osteopontin is an integral component of the CD44-ERM complex involved in cell migration. J Cell Physiol 184: 118-130, 2000.

23 Mi Z, Guo H, Wai PY, Gao C and Kuo PC: Integrin-linked kinase regulates osteopontin-dependent MMP-2 and uPA expression to convey metastatic function in murine mammary epithelial cancer cells. Carcinogenesis 27: 1134-1145, 2006.

24 Noti JD: Adherence to osteopontin via alphavbeta3 suppresses phorbol ester-mediated apoptosis in MCF-7 breast cancer cells that overexpress protein kinase C-alpha. Int J Oncol 17: 1237$1243,2000$.

25 Demirkan B: The Roles of Epithelial-to-Mesenchymal Transition (EMT) and Mesenchymal-to-Epithelial Transition (MET) in Breast Cancer Bone Metastasis: Potential Targets for Prevention and Treatment. J Clin Med 2: 264-282, 2013.

26 McCabe NP, De S, Vasanji A, Brainard J and Byzova TV: Prostate cancer specific integrin alphavbeta3 modulates bone metastatic growth and tissue remodeling. Oncogene 26: 62386243, 2007. 
27 Karlikova M, Topolcan O, Narsanska A, Kucera R, Treskova I and Treska V: Circulating Growth and Angiogenic Factors and Lymph Node Status in Early-stage Breast Cancer - A Pilot Study. Anticancer Res 36: 4209-4214, 2016.

28 Duarte-Salles T, Misra S, Stepien M, Plymoth A, Muller D, Overvad K, Olsen A, Tjønneland A, Baglietto L, Severi G, Boutron-Ruault MC, Turzanski-Fortner R, Kaaks R, Boeing H, Aleksandrova K, Trichopoulou A, Lagiou P, Bamia C, Pala V, Palli D, Mattiello A, Tumino R, Naccarati A, Bueno-de-Mesquita HB, Peeters PH, Weiderpass E, Quirós JR, Agudo A, SánchezCantalejo E, Ardanaz E, Gavrila D, Dorronsoro M, Werner M, Hemmingsson O, Ohlsson B, Sjöberg K, Wareham NJ, Khaw KT, Bradbury KE, Gunter MJ, Cross AJ, Riboli E, Jenab M, Hainaut $\mathrm{P}$ and Beretta L: Circulating Osteopontin and Prediction of Hepatocellular Carcinoma Development in a Large European Population. Cancer Prev Res (Phila) 9: 758-765, 2016.

29 Martinetti A, Miceli R, Sottotetti E, Di Bartolomeo M, de Braud F, Gevorgyan A, Dotti KF, Bajetta E, Campiglio M, Bianchi F, Bregni $G$ and Pietrantonio F: Circulating biomarkers in advanced colorectal cancer patients randomly assigned to three bevacizumab-based regimens. Cancers (Basel) 6: 1753-1768, 2014.

30 Polat B, Kaiser P, Wohlleben G, Gehrke T, Scherzad A, Scheich M, Malzahn U, Fischer T, Vordermark D and Flentje M: Perioperative changes in osteopontin and TGF $\beta 1$ plasma levels and their prognostic impact for radiotherapy in head and neck cancer. BMC Cancer 17: 6, 2017.

31 Živný JH, Leahomschi S, Klener P Jr., Živný J, Haluzík M and Cibula D: Comparison of Plasma Osteopontin Levels between Patients with Borderline Ovarian Tumours and Serous Ovarian Carcinoma. Folia Biol (Praha) 62: 258-262, 2016.
32 Lee YH and Song GG: Correlation between circulating osteopontin level in systemic lupus erythematosus and disease activity and associations between osteopontin polymorphisms and disease susceptibility: A meta-analysis. Lupus pii: 0961203316655214, in press, 2016.

33 Jakovac H, Kezele TG, Šućurović S, Mulac-Jeričević B and Radošević-Stašić B: Osteopontin-metallothionein I/II interactions in experimental autoimmunune encephalomyelitis. Neuroscience pii: S0306-4522(17)30178-1, 2017. doi: 10.1016/j.neuroscience. 2017.03.020.

34 Ostheimer C, Bache M, Güttler A, Reese T and Vordermark D: Prognostic information of serial plasma osteopontin measurement in radiotherapy of non-small-cell lung cancer. BMC Cancer 14: 858, 2014.

35 Kitagori K, Yoshifuji H, Oku T, Sasaki C, Miyata H, Mori KP, Nakajima T, Ohmura K, Kawabata D, Yukawa N, Imura Y, Murakami K, Nakashima R, Usui T, Fujii T, Sakai K, Yanagita M, Hirayama Y and Mimori T: Cleaved Form of Osteopontin in Urine as a Clinical Marker of Lupus Nephritis. PLoS One 11: e0167141, 2016. 